\title{
Helicobacter pylori-derived outer membrane vesicles stimulate interleukin 8 secretion through nuclear factor kappa B activation
}

\author{
Mun Sun Choi ${ }^{1}$, Eun Young Ze ${ }^{2}$, Jae Yong Park ${ }^{2}$, Tae-Seop Shin ${ }^{3}$, and Jae Gyu Kim²
}

${ }^{1}$ Division of Gastroenterology, Department of Internal Medicine, Armed Forces Capital Hospital, Seongnam; ${ }^{2}$ Department of Internal Medicine, Chung-Ang University College of Medicine, Seoul; ${ }^{3}$ Research Institute, Chung-Ang University, Seoul, Korea

Received: December 17, 2019

Revised : March 16, 2020

Accepted: March 19, 2020

\section{Correspondence to}

Jae Gyu Kim, M.D.

Department of Internal

Medicine, Chung-Ang University

College of Medicine, 102

Heukseok-ro, Dongjak-gu, Seoul

o6973, Korea

Tel: +82-2-6299-3147

Fax: +82-2-749-9150

E-mail:jgkimd@cau.ac.kr

https://orcid.org/0000-0002-

4841-9404
Background/Aims: Bacteria-derived outer membrane vesicles (OMVs) are commonly associated with various biological activities and functions. Helicobacter pylori-derived OMVs are thought to contribute to pathogenesis. This study aimed to investigate the effects of $\mathrm{H}$. pylori-derived OMVs.

Methods: $H$. pylori strains were isolated from patients with gastritis, gastric ulcer, or gastric cancer using endoscopic biopsy. The U-937, AGS, and MKN-45 cell lines were exposed to $H$. pylori and $H$. pylori-derived OMVs. The expression of interleukin 8 (IL-8) messenger RNA (mRNA) was assessed using reverse transcription-polymerase chain reaction (RT-PCR) and real-time RT-PCR, and IL-8 secretion was analyzed using enzyme-linked immunosorbent assay. Nuclear factor kappa B (NF-кB) activation was evaluated by Western blotting.

Results: $H$. pylori and $H$. pylori-derived OMVs induced the expression of IL-8 mRNA and protein. Importantly, the bacteria induced higher IL-8 mRNA and protein expression than the OMVs. IL-8 expression was induced to different levels in response to $\mathrm{H}$. pylori-derived OMVs from hosts with different gastric diseases. Western blotting revealed the increased phosphorylation and reduced degradation of inhibitor of NF- $\mathrm{KB}$ alpha in cells exposed to OMVs.

Conclusions: $H$. pylori-derived OMVs may aid the development of various gastric diseases by inducing IL-8 production and NF-кB activation.

Keywords: Helicobacter pylori; Extracellular vesicles; Bacterial outer membrane proteins; Interleukin-8; NF-kappa B

\section{INTRODUCTION}

Helicobacter pylori, gram-negative spiral bacteria, exhibit tropism toward the gastric epithelium; it is either found attached to the epithelial surface or within the mucus coating. Unlike most bacteria, $H$. pylori can survive and multiply in the gastric environment, which is known to be unfavorable for bacterial growth [1]. The remarkable characteristics of $H$. pylori that enable gastric colonization include microaerophilism, spiral shape with flagel- la, and urease activity to generate ammonium ions that buffer the gastric acids [2]. The mechanisms underlying H. pylori infection-induced tissue injury are not clearly established. Both bacterial and host factors seem to determine the outcomes $[3,4]$. Several studies on $H$. pylori virulence factors such as cytotoxin-associated gene A (CagA) and vacuolating cytotoxin A (VacA) proteins, urease, and host cell signaling pathways have helped elucidate the mechanism underlying $H$. pylori action $[5,6]$. However, several unresolved issues remain. 
Outer membrane vesicles (OMVs) released by several gram-negative bacteria contain numerous bacterial antigens and virulence factors. In addition, their release can be enhanced by antibiotic treatment $[7,8]$. Recent evidence suggests that OMVs play critical roles in the pathogenesis of bacterial infection, including those caused by $\mathrm{H}$. pylori, which has also been confirmed to secrete OMVs [9-11].

We hypothesized that OMVs secreted by $H$. pylori are important for the modulation of gastric epithelial cell functions related to the pathogenesis of $\mathrm{H}$. pylori-associated gastric diseases. This study aimed to evaluate the inflammatory responses induced in response to $H$. pylori-derived OMVs isolated from patients with gastritis, gastric ulcer, and gastric cancer.

\section{METHODS}

\section{H. pylori isolation}

Patients who underwent esophagogastroduodenoscopy and were diagnosed with gastritis, gastric ulcer, or gastric cancer at the Chung-Ang University Hospital, Seoul, Korea were included in the study. Six patients were enrolled, two patients for each disease type. H. pylori strains were isolated from gastric antral mucosal biopsy specimens collected from the enrolled patients. The study was approved by the Institutional Review Board of Chung-Ang University Hospital (10-080-12-23). Written informed consent was obtained from all study subjects.

\section{Cell lines and cell culture}

Two human gastric epithelial cell lines (AGS and MKN45) and a human monocytic cell line (U-937) were cultured in Roswell Park Memorial Institute (RPMI) 1680 medium supplemented with 10\% fetal bovine serum. Cell monolayers were cultured in petri dishes at $37.5^{\circ} \mathrm{C}$ in a humidified atmosphere containing $5 \% \mathrm{CO}_{2}$.

$H$. pylori strains were cultured under microaerophilic conditions $\left(37^{\circ} \mathrm{C}, 5 \% \mathrm{O}_{2}, 10 \% \mathrm{CO}_{2}, 85 \% \mathrm{~N}_{2}\right)$. In the $\mathrm{H}$. pylori group, the cells were infected with $\mathrm{H}$. pylori at a multiplicity of infection of 200:1 (bacterium-to-cell ratio of 200:1). In the OMV group, $10 \mathrm{ng} / \mathrm{mL}$ of OMVs, purified as mentioned below, were added. Before assessing the induction of inflammation in response to $\mathrm{H}$. pylori-derived OMVs in gastric epithelial cells, we conduct- ed a preliminary test using U-937, a monocyte cell line, to confirm whether OMVs could induce interleukin 8 (IL-8) secretion. U-937 cells were treated with different concentrations of OMVs (10, 100, or 1,000 ng/mL). Recombinant human IL-1 (PeproTech, Rocky Hill, NJ, USA) was used as the positive control.

\section{Preparation of OMVs}

H. pylori were cultured on blood agar plates for 72 hours in an atmosphere containing $10 \% \mathrm{CO}_{2}$. The cultured bacteria were resuspended in phosphate buffered saline (PBS). The resuspended $\mathrm{H}$. pylori was inoculated in 1-L Brain Heart Infusion liquid media containing 10\% horse serum, and cultured under microaerobic and shaking conditions until the optical density reached 1.o. The cultured bacteria were centrifuged twice for 15 minutes at 5,000 $\times g$ and $4^{\circ} \mathrm{C}$ to remove the $H$. pylori cells and the supernatant fraction was filtered using a $0.45-\mu \mathrm{m}$ vacuum filter. One liter of the filtrate was concentrated by ultrafiltration (QuixStand, GE Healthcare, Piscataway, NJ, USA; $100 \mathrm{kDa}$ hollow-fiber membrane) to a final volume of $50 \mathrm{~mL}$. The retentate was filtered again using a 0.22$\mu \mathrm{m}$ vacuum filter to remove any remaining cells. The resulting filtrate was subjected to ultracentrifugation (Optima XL-100K, Beckman, Brea, CA, USA) at 150,000 $\times g$ for 3 hours at $4^{\circ} \mathrm{C}$. The pellets were resuspended in 5 -mL PBS. Next, $15 \mathrm{~mL}$ of $30 \%$ iodixanol and $5 \mathrm{~mL}$ of $10 \%$ iodixanol solution were added consecutively in the 25 $\mathrm{mL}$ tube and centrifuged at $200,000 \times \mathrm{g}$ for 2 hours at $4^{\circ} \mathrm{C}$ and the whole fraction appearing between the PBS and $30 \%$ iodixanol layers was harvested. The fraction was resuspended in $25-\mathrm{mL}$ PBS and centrifuged at 150,000 $\times \mathrm{g}$ for 3 hours at $4^{\circ} \mathrm{C}$. The resultant pellet was resuspended in $0.2 \mathrm{~mL}$ of PBS. Purified OMVs were quantified using the Bradford dye assay (Bio-Rad Laboratories, Hercules, CA, USA) and stored at $-72^{\circ} \mathrm{C}$ until use. This protocol of OMV purification was generously provided by Professor YG Kim (Department of Life Science, Pohang University of Science and Technology) [12].

\section{Transmission electron microscopy and dynamic light scattering}

For transmission electron microscopy (TEM) studies, the OMVs were diluted to a concentration of $50 \mu \mathrm{g} / \mathrm{mL}$ using PBS and $10 \mathrm{~mL}$ of the mixture was placed on 300-mesh copper grids (EMS, Hatfield, PA, USA). Next, it was stained 
with $2 \%$ uranyl acetate. We imaged OMVs using the JEM1011 electron microscope (JEOL, Eching, München, Germany). For dynamic light scattering experiments, the OMVs were diluted to a concentration of $5 \mu \mathrm{g} / \mathrm{mL}$ in PBS, and $1 \mathrm{~mL}$ of the mixture was added into the cuvette. The diameters of the vesicles were measured using the Zetasizer Nano ZS (Malvern Instruments, Worcestershire, UK). The data were analyzed using the Dynamic V6 software (Protein Solutions, Lakewood, NJ, USA).

\section{RNA extraction}

RNA was extracted from each cell line that had been stimulated with $H$. pylori (bacterium-to-cell ratio of 200:1), OMVs (10, 100, or 1,000 ng/mL), and IL-1 (positive control). We used IL-1 as a positive control as it is known to be a positive regulator of IL-8 expression [13]. The RNA extracted from the untreated cells was used as a control. Total cellular RNA was extracted using an RNeasy mini kit (Qiagen, Valencia, CA, USA) and was treated with RNase-free DNase to remove any contaminating genomic DNA. The RNA pellet obtained was resuspended in $30 \mu \mathrm{L}$ of diethyl pyrocarbonate-treated water and stored at $-75^{\circ} \mathrm{C}$.

\section{Reverse transcription-polymerase chain reaction}

For reverse transcription-polymerase chain reaction (RT-PCR), $1 \mu \mathrm{g}$ of the total cellular RNA was reverse transcribed into complementary DNA (cDNA) using oligo dT primers, SuperScript ${ }^{\circledR}$ II Reverse Transcriptase (Invitrogen, Carlsbad, CA, USA; 50 units; $4 \mu \mathrm{L}$ ), and 2-mM dNTP, followed by incubation at $37^{\circ} \mathrm{C}$ for 70 minutes. Subsequently, the reaction mixture was heat inactivated for 10 minutes at $85^{\circ} \mathrm{C}$. The primers for $\beta$-actin and IL- $\delta$ were as follows (Bioneer, Daedeok, Daejeon, Korea): $\beta$-actin sense primer 5'-TGACGGGGTCACCCACACTGTGCCCATCTA-3' and $\beta$-actin antisense primer 5'-CTAGAAGCATTGCGGTGGACGATGGAGGG-3'; IL-8 sense primer 5'-ATGACTTCCAAGCTGGCCGTGGCT-3' and IL- 8 antisense primer 5'-TCTCAGCCCTCTTCAAAAACTTCTC-3'. After a hot start, the amplification program for $\beta$-actin was as follows: 45 -second denaturation at $95^{\circ} \mathrm{C}$ and 45 -second annealing and extension at $72^{\circ} \mathrm{C}$ for 25 cycles; the amplification program for IL- $\delta$ was as follows: 60 -second denaturation at $95^{\circ} \mathrm{C}$ and 2.5 -minute annealing and extension at $60^{\circ} \mathrm{C}$ for 35 cycles. Samples (10 $\mu \mathrm{L}$ ) of each amplified product were electrophoresed on a 1\% agarose gel.

\section{Real-time RT-PCR}

For real-time RT-PCR, $1 \mu \mathrm{L}$ of the cDNA was amplified on a 7300 real-time RT-PCR system (Applied Biosyste$\mathrm{ms}$, Foster City, CA, USA) using $10 \mu \mathrm{L} 2 \mathrm{x}$ SYBR Green master mix (Qiagen GmbH, Hilden, Germany) and a sample $(25 \mu \mathrm{L})$ of each amplified product, including 10 $\mathrm{pM}$ of primer. DNA amplification was performed using 40 cycles of $95^{\circ} \mathrm{C}$ for 15 seconds, followed by $60^{\circ} \mathrm{C}$ for 60 seconds. The $\beta$-actin primers included the sense primer 5'-AAGATGACCCAGATCATGT'T-3' and the antisense primer 5'-GCGACATAGCACAGCTTCT-3', and the IL- 8 primers included the sense primer 5'-ACATGACTTCCAAGCTGGCC- 3 ' and the antisense primer 5'-CAGAAATCAGGAAGGCTGCC-3'. All experiments were performed in triplicate and repeated thrice.

\section{Enzyme-linked immunosorbent assay}

The quantity of IL-8 secreted into the cell culture medium was measured using enzyme-linked immunosorbent assay (ELISA) with a monoclonal anti-human IL-8 antibody (Endogen, Woburn, MA, USA) for capture. This antibody was coated onto a flat-bottomed 96well NUNC-immuno plate (Nalge Nunc International, Roskilde, Denmark) and incubated overnight at room temperature and was subsequently cultured for 2 hours with a blocking buffer and then washed thrice. After washing, a biotin-labeled detecting antibody and horseradish peroxidase (HRP)-conjugated streptavidin were cultured sequentially for an hour, and then the plate was washed thrice. Tetramethylbenzidine substrate solution was added, followed by culturing for 30 minutes at room temperature. The response was terminated using 0.18 $\mathrm{M} \mathrm{H}_{2} \mathrm{SO}_{4}$. The IL-8 content was estimated by measuring absorbance at $450 \mathrm{~nm}$ using an ELISA reader and was expressed in $\mathrm{pg} / \mathrm{mL}$. All experiments were performed in triplicate and repeated thrice.

\section{Western blotting}

To determine the nuclear factor kappa B (NF- $\mathrm{B}$ ) activ-

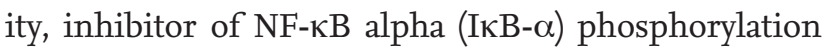
and degradation in the AGS cell line were assessed using Western blotting. The proteins were extracted from the AGS cells that had been stimulated with $10 \mu \mathrm{g}$ of $\mathrm{H}$. pylori-derived OMVs from each patient in a time-depen- 


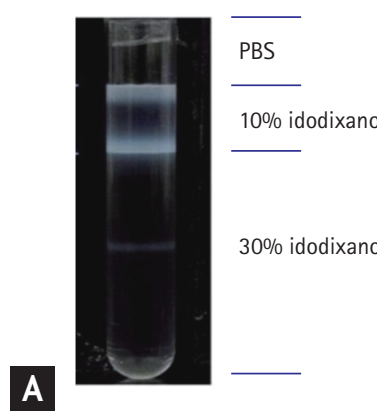

B
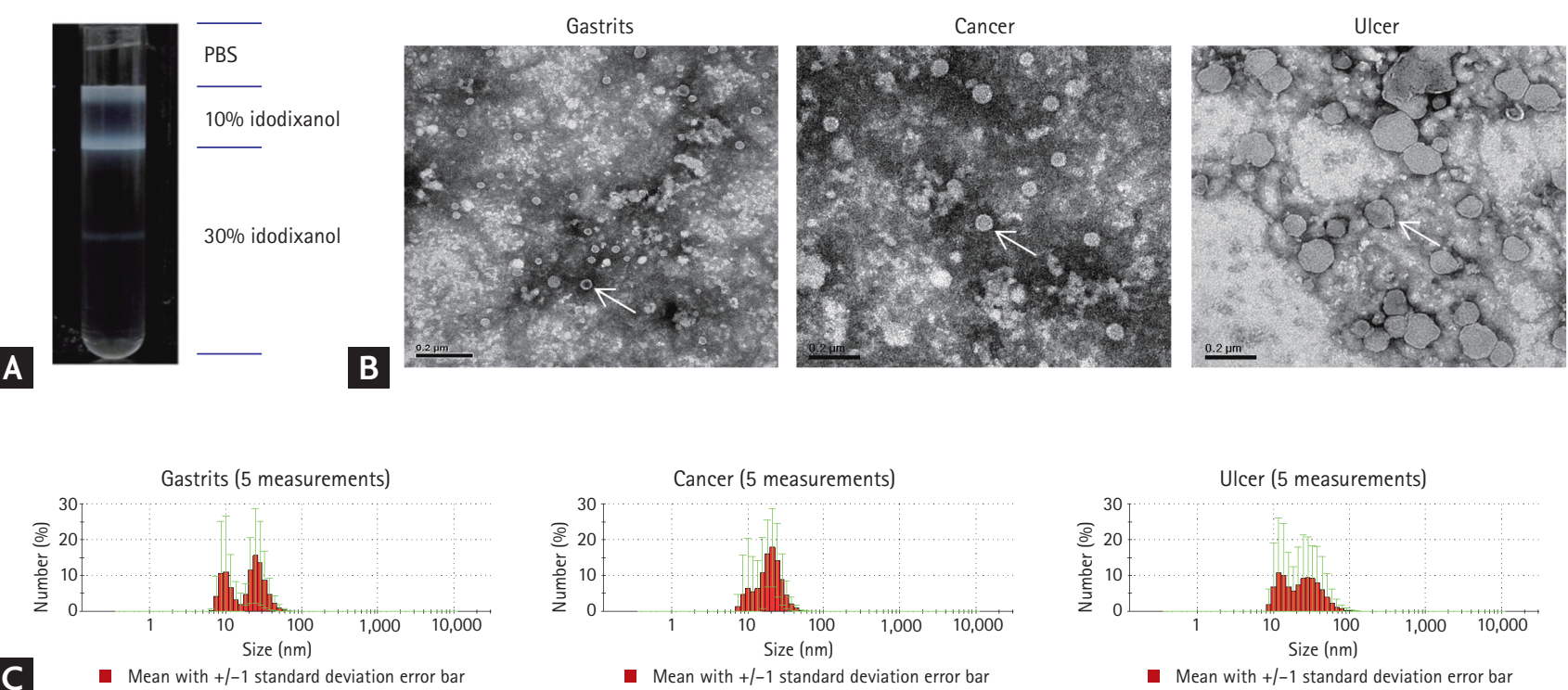

Figure 1. Helicobacter pylori-derived outer membrane vesicles (OMVs). (A) Isolation of $H$. pylori-derived OMVs using density gradient ultracentrifugation. The fractions were separated with phosphate buffered saline (PBS), and 10\% and 30\% iodixanol. (B) Transmission electron microscopy studies identified OMVs derived from H. pylori strains obtained from patients with gastritis, gastric cancer, or gastric ulcer. The arrows indicate the OMVs. (C) Dynamic light scattering revealed the size of OMVs from $H$. pylori strains obtained from patients with gastritis, gastric cancer, or gastric ulcer. .

dent manner. After harvesting, the cells were lysed with RIPA buffer and were centrifuged at $4^{\circ} \mathrm{C}$ for 30 minutes and $15,000 \times g$, following which the supernatant was harvested. The extracted proteins were quantified using the Bradford dye assay (Bio-Rad Laboratories) and boiled at $100^{\circ} \mathrm{C}$ for 5 minutes with $5 \times$ sodium dodecyl sulfate-polyacrylamide gel electrophoresis (SDS-PAGE) buffer. After separation at $80 \mathrm{~V}$ in stacking gel and at $100 \mathrm{~V}$ in separation gel, the samples were transferred to a Nitrocellulose membrane (Whatman GmbH, Dassel, Germany). Nonspecific binding was prevented by blocking with $5 \%$ non-fat milk for 2 hours at room temperature. Primary antibodies, anti-IкB- $\alpha$ antibody (Santa Cruz Biotech, Dallas, TX, USA), and antiphospho-IкB- $\alpha$ antibody (Santa Cruz Biotech) diluted with 5\% non-fat milk, were used to probe the membranes. After incubation, the membranes were washed thrice with PBS containing $0.05 \%$ Tween-20. The secondary antibody, anti-mouse IgG HRP (Santa Cruz Biotech), was diluted with $5 \%$ non-fat milk and used to probe the membrane for 2 hours. Following three washes with PBS, the protein bands were visualized by enhanced chemiluminescence (Santa Cruz Biotech) using the Bio-Imaging LAS 1000 plus (Fugifilm, Tokyo, Japan). Next, the Im-
ageJ software version 1.47 (National Institutes of Health, Bethesda, MD, USA) was used to quantify the results of Western blotting.

\section{Statistical analysis}

The expression of the IL-8 mRNA and protein is expressed as mean \pm standard error of the mean. Continuous variables were compared using the Mann-Whitney $U$ test. The Kruskal-Wallis test was used to evaluate the differences among three groups. Statistical significance was defined by $p<0.05$. Statistical analysis was performed using the SPSS software version 15.0 (SPSS Inc., Chicago, IL, USA).

\section{RESULTS}

\section{OMV isolation from $H$. pylori}

$H$. pylori-derived OMVs were separated using density gradients and centrifugation. The OMVs were detected in two particle bands, one band appeared between the PBS and 10\% iodixanol layers, and the other appeared between the $10 \%$ and $30 \%$ iodixanol layers (Fig. 1A). TEM studies identified that round to ovoid-shaped 
vesicles were derived from $H$. pylori in all specimens regardless of the underlying gastric disease (Fig. 1B). The vesicle size was approximately 10 to $100 \mathrm{~nm}$ regardless of the underlying gastric disease (Fig. $1 \mathrm{C}$ ).

\section{Induction of IL-8 mRNA expression in U-937, AGS, and MKN-45 cell lines by $H$. pylori-derived OMVs}

First, induction of IL-8 mRNA expression in the U-937 cell line in response to different quantities of $\mathrm{H}$. pylori-derived OMVs (10, 100, or 1,000 ng) was analyzed using RT-PCR and real-time RT-PCR. The expression of IL-8 mRNA was observed in response to $10 \mathrm{ng}$ or higher amounts of OMVs (Fig. 2A). As the quantity of OMVs increased, the IL- $S$ expression also increased in a dose-dependent manner (Fig. 2B). In a follow-up study, we determined that a dose of $10 \mathrm{ng}$ OMVs was adequate to induce a significant increase in IL- 8 mRNA expression.

As shown in Fig. 3A, IL-8 mRNA expression increased in the U-937 cell line upon exposure to $H$. pylori or OMVs (10 ng). IL-S mRNA expression was higher in cells exposed to $H$. pylori than that in cells exposed to OMVs. Conventional RT-PCR revealed that vesicles derived from $H$. pylori in patients with gastric cancer or gastric ulcer may induce higher level of IL- $S$ mRNA expression than those from patients with gastritis. However, real-time RT-PCR did not reveal any significant difference in the IL- 8 mRNA expression in U-937 cells when the cells were exposed to $H$. pylori derived from patients with different underlying gastric diseases $(p=0.874)$. In contrast, the IL- $\delta$ mRNA expression was significantly higher in cells exposed to OMVs from gastric cancer patient-derived strains than in those exposed to OMVs from gastritis patient-derived strains $(p=0.021)$.

IL- 8 mRNA expression also increased in AGS cells exposed to either H. pylori or to OMVs (10 ng). The expression was higher in $H$. pylori-exposed cells than that in the OMV-exposed cells. The expression of IL- 8 mRNA did not differ based on the underlying gastric disease in AGS cells exposed to bacteria $(p=0.626)$ or OMVs $(p=$ 0.122) (Fig. 3B).

Similar results were observed in the MKN-45 cell line exposed to either H. pylori or OMVs (10 ng). The expression of IL- 8 mRNA was also higher in cells exposed to the bacteria than in those exposed to OMVs. In the cells exposed to either bacteria or OMVs, IL-S mRNA expression did not differ based on the underlying gastric dis-

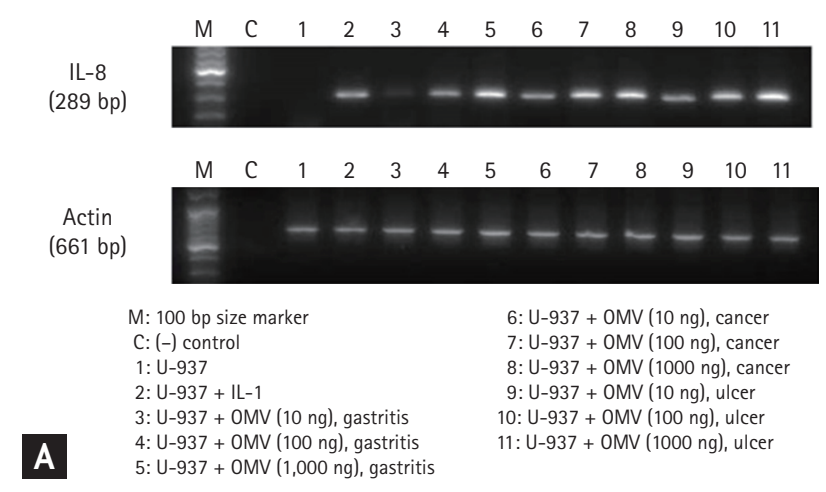

B

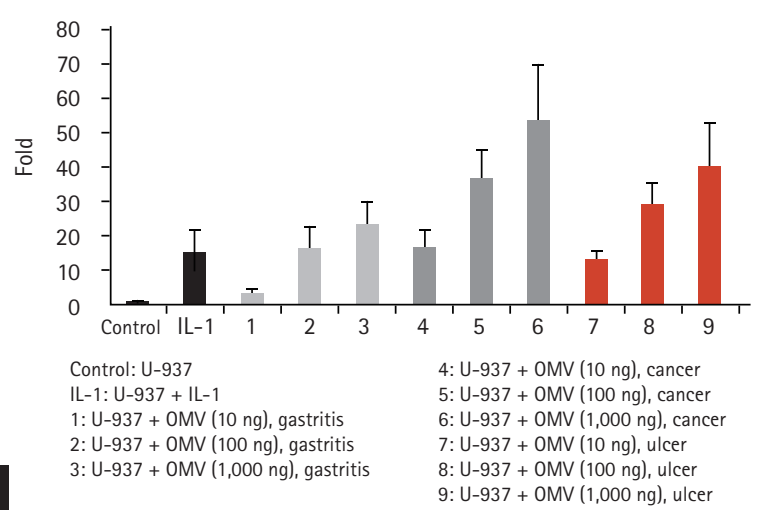

Figure 2. Helicobacter pylori-derived outer membrane vesicles (OMVs) and interleukin 8 (IL-S) messenger RNA (mRNA) expression in the U-937 cell line. (A) Reverse transcription-polymerase chain reaction (RT-PCR) studies showed that OMVs from $H$. pylori strains stimulate IL- 8 mRNA expression. (B) Real-time quantitative RT-PCR showed that a higher quantity of OMVs was dose-dependently associated with the higher expression of IL-8 mRNA.

ease (bacteria, $p=0.995 ;$ OMVs, $p=0.241$ ) (Fig. $3 \mathrm{C}$ ).

\section{Stimulation of IL-8 secretion by $H$. pylori-derived OMVs}

Enhanced IL-8 production in the U-937 cell line exposed to $H$. pylori and OMVs (10 ng) was detected using ELISA. IL-8 secretion was higher in cells exposed to bacteria than in those exposed to OMVs. IL-8 production in cells exposed to bacteria was highest in case of gastric ulcer-patient-derived strains, followed by gastric cancer patient- and gastritis patient-derived strains $(p=0.001)$. ELISA also revealed that the IL-8 levels were higher in cells exposed to OMVs from gastric cancer or gastric ulcer patient-derived strains than in those exposed to OMVs from gastritis patient-derived strains $(p=0.018)$ (Fig. $4 \mathrm{~A})$. 
(a)

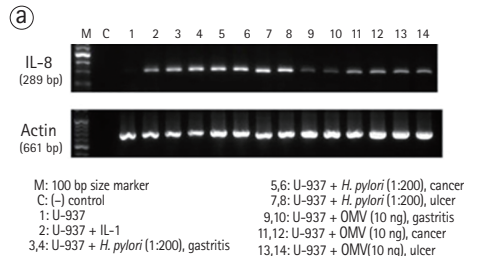

(b)

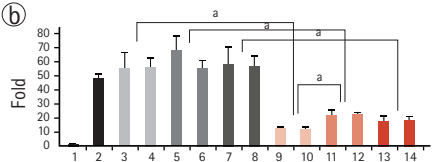

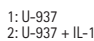

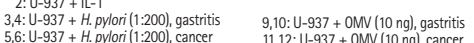

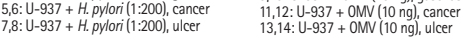

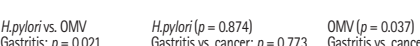

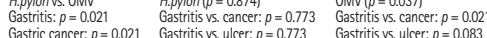
A (a)

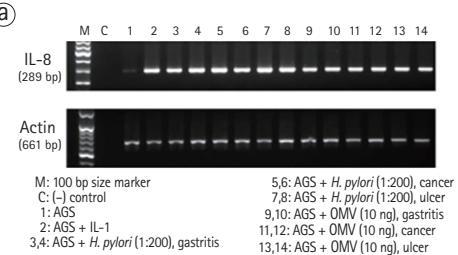

(b)

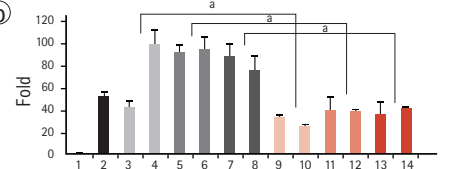

1:AGS
2:AGS $A-1-1$

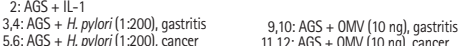

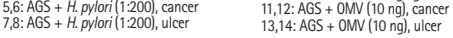

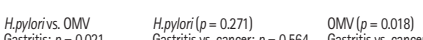

$\begin{array}{lll}\text { Gastrititis: } p=0.021 & \text { Gastritis vs. cancer: } p=0.564 \quad \text { Gastritis vs. cancer: } p=0.0 .083\end{array}$ B $\begin{array}{lll}\text { Gastric cancer: } p=0.021 & \text { Gastritis vs. ulcer: } p=0.564 & \text { Gastritis vs. ulcer: } p=0.081 \\ \text { Gastric ulcer: } p=0.020 & \text { Cancer vs. ulcer: } p=0.309 & \text { Cancer vs. ulcer: } p=0.772\end{array}$ (a)

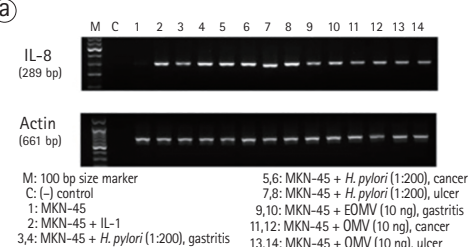

(b)

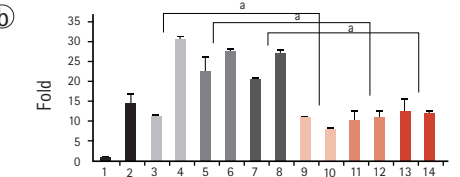

1: $M K N-45$

3.4: MKN-45 + H. pylori (1:200), gastritis $\quad 9,10:$ MKN- $45+$ OMV $(10 \mathrm{ng})$, gastritis

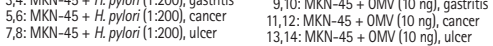

H.pylorivs. OMV H.pylori $(p=0.995) \quad \operatorname{OMV}(p=0.241)$ $\begin{array}{lll}\text { Gastritis: } p=0.020 & \text { Gastritis v5. cancer: } p=1.000 \quad \text { Gastrititivs cancer: } p=0.384 \\ \text { Gastric cancer: } p=0.021 & \text { Gastritis vs ulcer: } p=1.000 & \text { Gastritis vs ulcer: } p=0.339\end{array}$ $\begin{array}{lll}\text { Gastric cancer: } p=0.021 & \text { Gastrititi vs ulcer: } p=1.000 & \text { Gastrititis vs ulcer: } p=0.139 \\ \text { Gastric ulcer: } p=0.020 & \text { Cancer vs. ulcer: } p=0.884 & \text { Cancer vs. ulcer: } p=0.248\end{array}$

Figure 3. Interleukin 8 (IL-S) messenger RNA (mRNA) expression in U-937, AGS, and MKN-45 cells exposed to Helicobacter pylori bacteria or outer membrane vesicles (OMVs). (A) IL- 8 mRNA expression in U-937 cells exposed to $H$. pylori bacteria or OMVs. (a) Polymerase chain reaction (PCR) amplification. (b) Real-time quantitative reverse transcription PCR (RT-PCR). (B) IL- 8 mRNA expression in AGS cells exposed to H. pylori bacteria and OMVs. (a) PCR amplification. (b) Real-time quantitative RT-PCR. (C) IL-S mRNA expression in MKN-45 cells exposed to H. pylori bacteria and OMVs. (a) PCR amplification. (b) Real-time quantitative RT-PCR. H. pylori bacteria and OMVs were obtained from six patients (two patients each of gastritis, gastric cancer, and gastric ulcer). ${ }^{\mathrm{a}} \mathrm{p}<0.05$.

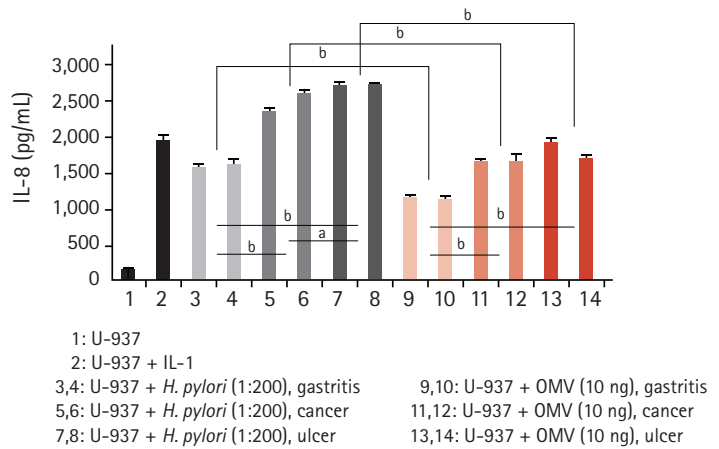
13,14: U-937 + OMV (10 ng), ulcer

\section{H.pylori $(p=0.001)$}

$\operatorname{OMV}(p=0.018)$ Gastritis: $p=0.004 \quad$ Gastritis vs. cancer: $p=0.004$

Gastric cancer: $p=0.004$ Gastritis vs. ulcer: $p=0.004$ Gastric ulcer: $p=0.004 \quad$ Cancer vs. ulcer: $p=0.037$

Gastritis vs, cancer: $p=0.00$

Gastritis vs. cancer: $p=0.004$ Cancer vs. ulcer: $p=0.109$

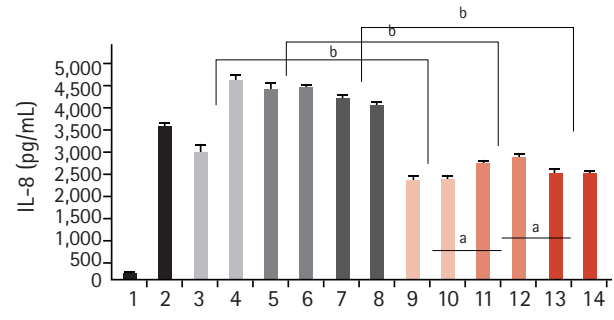

1: AGS

2: AGS + IL-1

3,4: AGS + H. pylori $(1: 200)$, gastritis $\quad 9,10: A G S+O M V(10 \mathrm{ng})$, gastritis

5,6: AGS + H. pylori (1:200), cancer 11,12: AGS + OMV $(10 \mathrm{ng})$, cancer

7,8: AGS + H. pylori (1:200), ulcer $\quad 13,14: \mathrm{AGS}+\mathrm{OMV}(10 \mathrm{ng})$, ulcer Gastritis: $p=0.006$

B Gastritis vs. cancer: $p=0.423$ Gastritis vs. cancer: $p=0.010$ Gastric cancer: $p=0.004 \quad$ Gastritis vs. ulcer: $p=0.873 \quad$ Gastritis vs. ulcer: $p=0.336$ Gastric ulcer: $p=0.004 \quad$ Cancer vs. ulcer: $p=0.055 \quad$ Cancer vs. ulcer: $p=0.037$

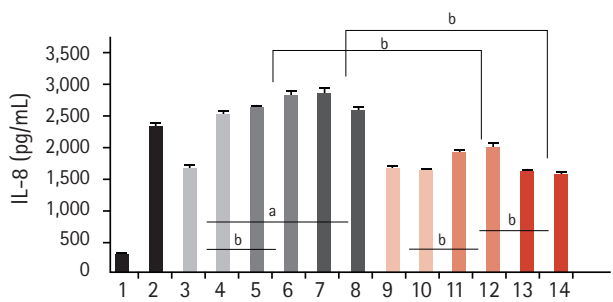

1: MKN-45

2: MKN-45 + IL-1

3,4: MKN-45 + H. pylori $(1: 200)$, gastritis 5,6: MKN-45 + H. pylori (1:200), cancer 7,8: MKN-45 + H. pylori ( $1: 200)$, ulcer

9,10: MKN-45 + OMV (10 ng), gastritis 11,12: MKN-45 + OMV (10 ng), cancer 13,14: MKN-45 + OMV (10 ng), ulcer
H.pylorivs. OMV Gastritis: $p=0.109$
H.pylori $(p=0.011)$

$\begin{array}{ll}\text { H.pylori }(p=0.011) & \mathrm{OMV}(p=0.002) \\ \text { Gastritis vs. cancer: } p=0.006 & \text { Gastritis vs. cancer: }\end{array}$ Gastric cancer: $p=0.004 \quad$ Gastritis vs. ulcer: $p=0.016$ Gastric ulcer: $p=0.004 \quad$ Cancer vs. ulcer: $p=1.000$
Gastritis vs. cancer: $p=0.004$ Gastritis vs. ulcer: $p=0.173$ Cancer vs. ulcer: $p=0.004$
Figure 4. Interleukin 8 (IL-8) secretion in U-937, AGS, and MKN-45 cells exposed to Helicobacter pylori bacteria or outer membrane vesicles (OMVs). (A) In the U-937 cells, the IL-8 levels were quantified using enzyme-linked immunosorbent assay (ELISA). (B) In the AGS cells, the IL-8 levels were quantified using ELISA. (C) In the MKN-45 cells, the IL-8 levels were quantified using ELISA. $H$. pylori and OMVs were obtained from six patients (two patients each of gastritis, gastric cancer, and gastric ulcer). The data are presented as mean \pm standard errors for three experiments. ${ }^{\mathrm{a}} \mathrm{p}<0.05,{ }^{\mathrm{b}} \mathrm{p}<0.01$. 
IL-8 levels also increased in AGS cells exposed to either H. pylori or OMVs (10 ng). The expression was stronger in the H. pylori-exposed cells than that in the OMV-exposed cells. The IL-8 levels in H. pylori-exposed cells did not vary significantly based on the associated gastric disease $(p=0.271)$. However, in case of cells exposed to OMVs, OMVs derived from gastric cancer patient-derived strains induced higher levels of IL-8 production than did those derived from gastric ulcer or gastritis patient-derived strains $(p=0.018)$ (Fig. $4 \mathrm{~B}$ ).

As shown in Fig. 4C, the IL-8 levels also increased in the MKN-45 cell line exposed to either $H$. pylori or OMVs (10 ng). The IL- 8 mRNA expression levels were higher in cells exposed to bacteria than in those exposed to OMVs derived from patients with gastric cancer or gastric ulcer. Moreover, the bacteria derived from gastric cancer

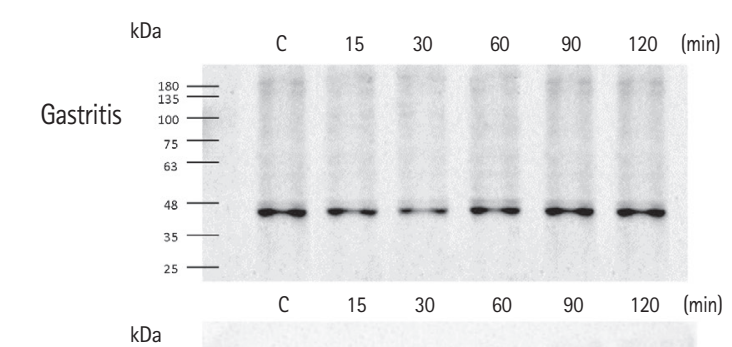

A
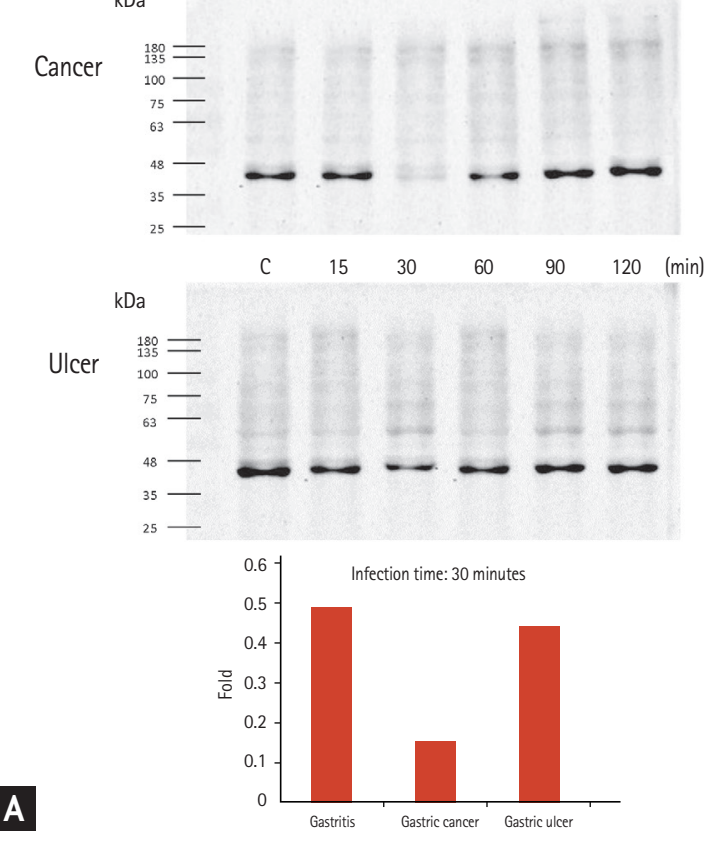

or gastric ulcer patients tended to induce higher IL-8 expression than those from gastritis patients $(p=0.011)$. Among the OMV-exposed cells, the vesicles from gastric cancer patient-derived strains induced higher levels of IL-8 expression than those from gastric ulcer or gastritis patient-derived strains $(p=0.002)$. In all three cell lines, the OMVs from gastric cancer patient-derived strains tended to induce higher levels of IL-8 expression than those from gastritis patient-derived strains.

\section{NF- $\kappa$ B production activated by $H$. pylori-derived OMVs}

Western blotting revealed that IкB- $\alpha$ degradation reduced significantly, while IкB- $\alpha$ phosphorylation increased significantly 30 minutes after the AGS cells were exposed to $H$. pylori-derived OMVs. In particular, IкB- $\alpha$

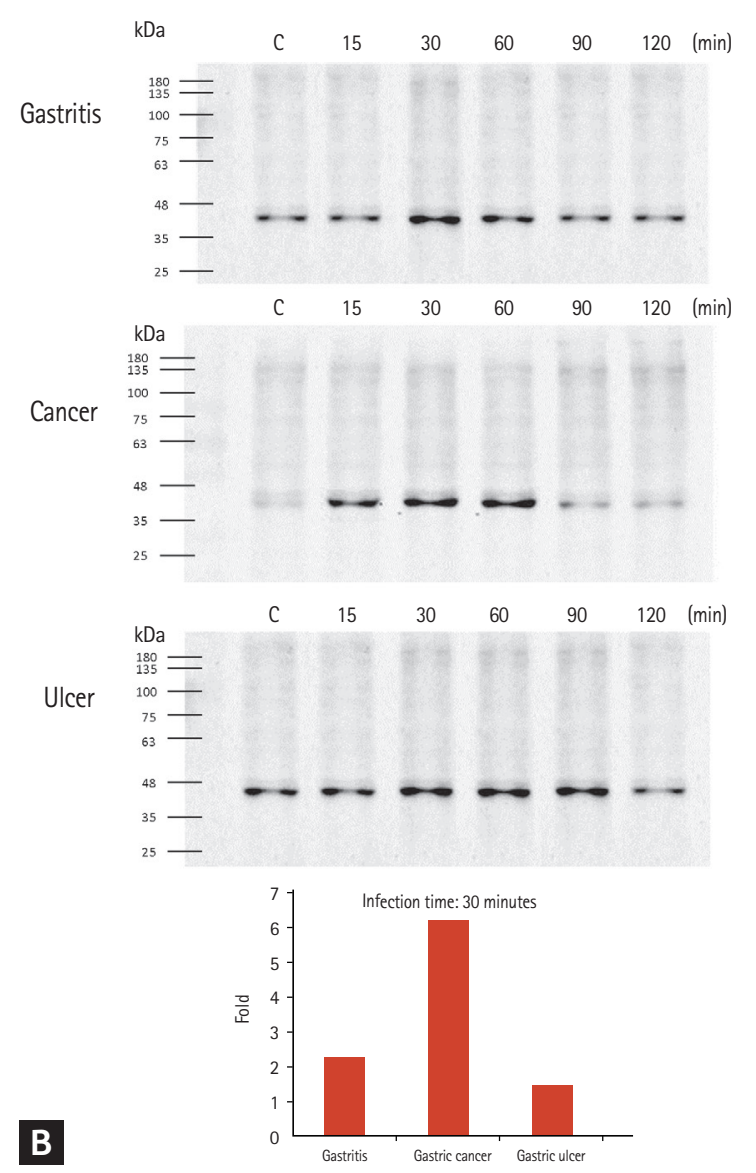

Figure 5. Inhibitor of nuclear factor kappa B, alpha $\left(\mathrm{I}_{\kappa} \mathrm{B}-\alpha\right)$ degradation and phosphorylation in AGS cells analyzed by Western blotting after inoculation with Helicobacter pylori-derived outer membrane vesicles (OMVs). (A) IאB- $\alpha$ degradation was analyzed using Western blotting. The AGS cells were treated with $\mathrm{H}$. pylori-derived OMVs (10 ng) obtained from each patient. (B) The phosphorylation of IкB- $\alpha$ was analyzed by Western blotting in a time-dependent manner. The AGS cells were treated with $H$. pylori-derived OMVs (10 ng) obtained from each patient. C, control. 
degradation was significantly reduced (by o.2-fold) in the cells treated with OMVs from gastric cancer patient-derived strains compared with that in the control (o-minute) (Fig. 5A). IкB- $\alpha$ phosphorylation was significantly enhanced (by 6.2-fold) in cells treated with OMVs from gastric cancer patient-derived strains compared with that in the control (o-minute) (Fig. $\left.{ }_{5} \mathrm{~B}\right)$.

\section{DISCUSSION}

Recently, OMVs from $H$. pylori have been identified to play a pivotal role in the pathogenesis of $H$. pylori infection. H. pylori-derived OMVs are small, circular structures with intact outer membranes that are shed from the surface of $H$. pylori cells. In this study, we isolated $H$. pylori-derived OMVs from patients with different gastric diseases. The size or appearance of OMVs among the different disease groups differed from each other. We proposed that OMVs from $H$. pylori can modulate the function of gastric epithelial cells. To investigate this, we studied the effects of $H$. pylori-derived OMVs on IL-S mRNA expression, IL-8 secretion, and NF- $\kappa \mathrm{B}$ activation in gastric epithelial cell lines and in a monocytic cell line. IL-8 appeared to play a crucial role in the recruitment and activation of neutrophils in the gastric mucosa [14]. Previous reports have suggested that gastric epithelial cells are an important source of IL-8 [15,16]. Prolonged IL-8 production during $H$. pylori infection could result in leukocyte recruitment to infected tissues, which is an important step in the regulation of inflammatory and immune responses to $H$. pylori [17]. IL-8 expression is primarily controlled at the transcriptional level. The promoter region of IL- $\delta$ contains binding sites for the inducible transcription factors, NF- $\mathrm{KB}$ and activator protein-1 (AP-1) [18]. The transcriptional activity of NF- $\mathrm{\kappa B}$ is controlled by its cytoplasmic inhibitory protein IкB- $\alpha$ [19]. Previous studies have reported that IL-S transcription in response to $H$. pylori infection in MKN 45, Kato III, and AGS cells requires the activation of NF-KB, and to a lesser extent, AP-1 [20-22].

Coincubation of U-937, AGS, or MKN-45 cells with OMVs derived from $H$. pylori significantly increased IL- 8 mRNA expression and IL-8 secretion. The OMVs also activated NF- $\mathrm{\kappa B}$ in AGS cells. In this study, we demonstrated that $H$. pylori-derived OMVs directly af-

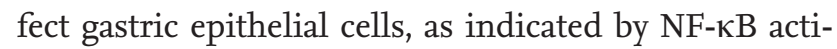
vation and IL-8 expression similar to that induced by bacteria. These observations support the hypothesis that the effect of $H$. pylori on gastric epithelial cells is partly dependent on bacterial OMVs. We have previously demonstrated that $H$. pylori-derived OMVs induce IL-8 expression via fusion with the AGS cells [13]. Additionally, the magnitude of IL-8 stimulation and expression was greater in AGS cells than that in the MKN-45 cells. Although both cells represent gastric epithelial cell lines, the differences in the response to OMVs in each cell line might be attributed to their different origin and different susceptibility to pathogen associated molecular patterns. This could also be partially predicted by the fact that the magnitude of response to IL-1, which was used as a positive control, was lesser in the $\mathrm{MKN}-45$ cells than that in the AGS cells.

OMVs are natural vehicles for the directed and specific intercellular transport of bacterial virulence factors. Vesicle surface factors can mediate adhesion to eukaryotic cells as well as the subsequent internalization of the vesicle contents [23]. The roles of OMVs in the pathogenesis of $H$. pylori infection are unclear. However, internalized $H$. pylori OMVs may transfer their toxins to the host epithelial cells, resulting in NF- $\kappa \mathrm{B}$ activation and IL-8 production $[13,24]$. Peptidoglycan (PG) is expectedly one of the most important virulence factors present in $\mathrm{H}$. pylori-derived OMVs. Regardless of the ability of gram-negative pathogens to invade host cells or to express a bacterial secretion system, their PG molecules can be detected by the nucleotide-binding oligomerization domain-containing protein 1 (NOD1), leading to the initiation of NOD1-dependent proinflammatory responses in host cells. $H$. pylori have previously been reported to transfer PG into the host epithelial cells through an unknown mechanism. In a recent study, OMVs released by $H$. pylori were found to enhance NF$\kappa \mathrm{B}$ and NOD1-dependent responses [25].

H. pylori OMVs also contain proteins, lipopolysaccharide (LPS), lipoproteins, and VacA; however, there is no evidence regarding the presence of CagA or urease [11]. H. pylori LPS has been shown to induce IL-8 expression in gastric epithelial cells [16]. Altered phosphorylation of lipid A in H. pylori LPS is generally considered to attenuate the toxicity of LPS as a virulence factor $[26,27]$. Recently, H. pylori LPS has been shown to activate NF-кB 
in guinea pig gastric cells via a toll-like receptor 4 (T'LR4) [28]. These observations suggest that LPS present within the vesicles could account for the pathogenicity of $H$. pylori OMVs.

Based on our findings, we can suggest that $H$. pylori OMVs may contribute to NF- $\kappa B$ activation and IL-8 production via NOD1 or the TLR4 response by transporting toxins such as PG or LPS. Additionally, each cell line responded differently to $H$. pylori cells and their respective OMVs. As shown in this study, IL-8 expression was higher in cells exposed to whole $H$. pylori bacteria than in those exposed to the OMVs. In fact, we had treated the AGS cells with $H$. pylori at a colony-forming unit (CFU):cell ratio of 200:1, and with OMVs at a pg:cell ratio of 1:5. When we calculated the equivalent dose of OMVs secreted by $1 \mathrm{CFU}$ of $\mathrm{H}$. pylori, the dose was $2 \mathrm{fg}$, suggesting that $200 \mathrm{CFUs}$ of $\mathrm{H}$. pylori secrete $0.4 \mathrm{pg}$ of OMVs. As a positive control, we treated $5 \times 10^{4}$ AGS cells with $1 \times 10^{7}$ CFUs of $H$. pylori, or with $10 \mathrm{ng}$ of OMVs. This implies that the quantity OMVs used to treat the AGS cells was half that of $\mathrm{H}$. pylori. However, another reason for the higher IL-8 expression in cells exposed to H. pylori could be that live bacteria may contain or generate a higher number and wider variety of virulence factors compared to OMVs, in addition to possessing several mechanisms to inject virulence factors into the host cells. Additionally, live $H$. pylori bacteria can secrete OMVs continuously in the culture medium. Therefore, it is challenging to suggest that OMVs are the sole contributors to H. pylori pathogenesis. Factors other than OMVs, such as CagA, may be responsible as well.

In spite of the increasing information about the mechanisms underlying $H$. pylori-induced pathogenesis, there is limited understanding of the reasons due to which infected individuals develop any one of the diseases. This lack of concrete evidence emphasizes the importance of multiple bacterial factors, interstrain differences, variable host responses to the bacteria, differences in host-microbe interactions, and differences in the genetic susceptibility of the hosts in determining the course and outcome of $H$. pylori infections [2931]. In this study, the expression of IL- 8 induced by $H$. pylori-derived OMVs from hosts with different gastric diseases differed from each other in monocytes (U-937 cells) or in gastric epithelial cells. We could infer that OMVs associated with different underlying gastric dis- eases have different potentials for inducing inflammatory responses in gastric epithelial cells. This difference in IL-8 expression might result from the differences in the virulence factors contained in $\mathrm{H}$. pylori-derived OMVs from hosts with different gastric diseases. This difference in vesicle phenotype might explain the various host inflammatory responses to $H$. pylori and the corresponding disease outcomes.

This study has several limitations. First, we used density gradient centrifugation to separate the lipid-containing vesicles from contaminating proteins and other structures in the cell-free supernatants. Several studies have demonstrated the effectiveness of this protocol in the purification of $H$. pylori OMVs $[9,11,32,33]$. However, there remains a possibility of residual lysate contamination of the vesicle preparations. Second, the variability in the size of OMVs might have impaired the vesicle purification and quantification process. We used $10 \mathrm{ng}$ of OMVs for the experiments in this study; however, it is possible that the quantities of OMVs varied marginally. Third, we did not analyze the differences in the expression pattern of virulence factors among $H$. pylori-derived OMVs from hosts with different gastric diseases. Additionally, the limited sample numbers make it difficult to draw conclusions about the different IL-8 responses and the disease outcomes among them. This study primarily focused on the clinical aspects, demonstrating the difference in the pathogenesis of $H$. pylori-derived OMVs from hosts with different gastric diseases. Besides, in a previous study, we showed that $H$. pylori-derived OMVs contain CagA or VacA [13]. To further clarify the relationship between the different virulence factors in OMVs and various disease outcomes, the mechanistic aspect of this finding should be investigated in future research.

In conclusion, we observed that OMVs shed from the surface of $H$. pylori exert a measurable effect on gastric epithelial cell responses, similar to those exerted by whole bacteria on the induction of IL-8 secretion and NF- $\kappa \mathrm{B}$ activation. $H$. pylori-derived OMVs may be involved in the pathogenesis of $H$. pylori-associated gastric disease. Although not all results were significant, the different responses induced by OMVs associated with different underlying diseases suggested that strains isolated from individuals with each disease type produce OMVs with distinct characteristics. Further research is warranted to establish the optimal immunotherapeutic 
strategy for the primary prevention of $H$. pylori-related gastrointestinal disease.

\section{KEY MESSAGE}

1. Outer membrane vesicles shed from the surface of Helicobacter pylori exert a measurable effect on gastric epithelial cell responses.

2. The induction of interleukin 8 (IL-8) production and nuclear factor kappa B (NF- $\mathrm{B})$ activation by $H$. pylori-derived outer membrane vesicles isolated from gastric cancer patients was more significant than that induced by $H$. pylori-derived outer membrane vesicles isolated from healthy controls and ulcer patients.

3. The different responses induced by $\mathrm{H}$. pylori-derived vesicles associated with different underlying diseases suggested that strains associated with each disease produce outer membrane vesicles with distinct characteristics.

\section{Conflict of interest}

No potential conflict of interest relevant to this article was reported.

\section{Acknowledgments}

This study was supported by the Basic Science Research Program through the National Research Foundation of Korea (NRF) funded by the Ministry of Education (NRF2012R1A1A2007865).

\section{REFERENCES}

1. Blaser MJ, Atherton JC. Helicobacter pylori persistence: biology and disease. J Clin Invest 2004;113:321-333.

2. Scott DR, Weeks D, Hong C, Postius S, Melchers K, Sachs $\mathrm{G}$. The role of internal urease in acid resistance of Helicobacter pylori. Gastroenterology 1998;114:58-70.

3. Kalisperati P, Spanou E, Pateras IS, et al. Inflammation, DNA damage, Helicobacter pylori and Gastric Tumorigenesis. Front Genet 2017;8:20.

4. Allen LA, Schlesinger LS, Kang B. Virulent strains of Helicobacter pylori demonstrate delayed phagocytosis and stimulate homotypic phagosome fusion in macrophages.
J Exp Med 2000;191:115-128.

5. Yeo M, Hahm KB. Perspective of Helicobacter pylori research: molecular pathogenesis of Helicobacter pylori virulence factors. Korean J Gastroenterol 2005;46:181-188.

6. Backert S, Naumann M. What a disorder: proinflammatory signaling pathways induced by Helicobacter pylori. Trends Microbiol 2010;18:479-486.

7. Devos S, Van Oudenhove L, Stremersch S, et al. The effect of imipenem and diffusible signaling factors on the secretion of outer membrane vesicles and associated Ax21 proteins in Stenotrophomonas maltophilia. Front Microbiol 2015;6:298.

8. Maredia R, Devineni N, Lentz P, et al. Vesiculation from Pseudomonas aeruginosa under SOS. ScientificWorldJournal 2012;2012:402919.

9. Jan AT. Outer membrane vesicles (OMVs) of Gram-negative bacteria: a perspective update. Front Microbiol 2017;8:1053.

10. Hynes SO, Keenan JI, Ferris JA, Annuk H, Moran AP. Lewis epitopes on outer membrane vesicles of relevance to Helicobacter pylori pathogenesis. Helicobacter 2005;10:146-156.

11. Keenan J, Day T, Neal S, et al. A role for the bacterial outer membrane in the pathogenesis of Helicobacter pylori infection. FEMS Microbiol Lett 2000;182:259-264.

12. Lee EY, Bang JY, Park GW, et al. Global proteomic profiling of native outer membrane vesicles derived from Escherichia coli. Proteomics 2007;7:3143-3153.

13. Choi HI, Choi JP, Seo J, et al. Helicobacter pylori-derived extracellular vesicles increased in the gastric juices of gastric adenocarcinoma patients and induced inflammation mainly via specific targeting of gastric epithelial cells. Exp Mol Med 2017;49:e330.

14. Peterson WL. Helicobacter pylori and peptic ulcer disease. N Engl J Med 1991;324:1043-1048.

15. O'Hara AM, Bhattacharyya A, Mifflin RC, et al. Interleukin-8 induction by Helicobacter pylori in gastric epithelial cells is dependent on apurinic/apyrimidinic endonuclease-1/redox factor-1. J Immunol 2006;177:7990-9.

16. Huang J, O'Toole PW, Doig P, Trust TJ. Stimulation of interleukin-8 production in epithelial cell lines by Helicobacter pylori. Infect Immun 1995;63:1732-1738.

17. Ando T, Kusugami K, Ohsuga M, et al. Interleukin-8 activity correlates with histological severity in Helicobacter pylori-associated antral gastritis. Am J Gastroenterol 1996;91:1150-1156. 
18. Roebuck KA. Oxidant stress regulation of IL-8 and ICAM1 gene expression: differential activation and binding of the transcription factors AP-1 and NF-kappaB (review). Int J Mol Med 1999;4:223-230.

19. Thanos D, Maniatis T. NF-kappa B: a lesson in family values. Cell 1995;80:529-532.

20. Aihara M, Tsuchimoto D, Takizawa H, et al. Mechanisms involved in Helicobacter pylori-induced interleukin-8 production by a gastric cancer cell line, MKN45. Infect Immun 1997;65:3218-3224.

21. Masamune A, Shimosegawa T, Masamune O, Mukaida N, Koizumi M, Toyota T. Helicobacter pylori-dependent ceramide production may mediate increased interleukin 8 expression in human gastric cancer cell lines. Gastroenterology 1999;116:1330-1341.

22. Chu SH, Kim H, Seo JY, Lim JW, Mukaida N, Kim KH. Role of NF-kappaB and AP-1 on Helicobater pylori-induced IL-8 expression in AGS cells. Dig Dis Sci 2003;48:257-265.

23. Kuehn MJ, Kesty NC. Bacterial outer membrane vesicles and the host-pathogen interaction. Genes Dev 2005;19:2645-2655.

24. Turner L, Bitto NJ, Steer DL, et al. Helicobacter pylori outer membrane vesicle size determines their mechanisms of host cell entry and protein content. Front Immunol 2018;9:1466.

25. Kaparakis M, Turnbull L, Carneiro L, et al. Bacterial membrane vesicles deliver peptidoglycan to NODı in ep- ithelial cells. Cell Microbiol 2010;12:372-385.

26. Chmiela M, Miszczyk E, Rudnicka K. Structural modifications of Helicobacter pylori lipopolysaccharide: an idea for how to live in peace. World J Gastroenterol 2014;20:9882-9897.

27. Moran AP, Helander IM, Kosunen TU. Compositional analysis of Helicobacter pylori rough-form lipopolysaccharides. J Bacteriol 1992;174:1370-1377.

28. Kawahara T, Kuwano Y, Teshima-Kondo S, et al. Toll-like receptor 4 regulates gastric pit cell responses to Helicobacter pylori infection. J Med Invest 2001;48:190-197.

29. Peek RM Jr, Blaser MJ. Helicobacter pylori and gastrointestinal tract adenocarcinomas. Nat Rev Cancer 2002;2:2837.

30. El-Omar EM, Carrington M, Chow WH, et al. The role of interleukin-1 polymorphisms in the pathogenesis of gastric cancer. Nature 2001;412:99.

31. Ernst PB, Gold BD. The disease spectrum of Helicobacter pylori: the immunopathogenesis of gastroduodenal ulcer and gastric cancer. Annu Rev Microbiol 2000;54:615-640.

32. Ismail S, Hampton MB, Keenan JI. Helicobacter pylori outer membrane vesicles modulate proliferation and interleukin-8 production by gastric epithelial cells. Infect Immun 2003;71:5670-5675.

33. Kesty NC, Mason KM, Reedy M, Miller SE, Kuehn MJ. Enterotoxigenic Escherichia coli vesicles target toxin delivery into mammalian cells. EMBO J 2004;23:4538-4549. 